\title{
A posteriori error estimator based on gradient recovery by averaging for discontinuous Galerkin methods
}

\author{
Emmanuel Creusé, Serge Nicaise ${ }^{\dagger}$
}

March 19, 2010

\begin{abstract}
We consider some (anisotropic and piecewise constant) diffusion problems in domains of $\mathbb{R}^{2}$, approximated by a discontinuous Galerkin method with polynomials of any fixed degree. We propose an a posteriori error estimator based on gradient recovery by averaging. It is shown that this estimator gives rise to an upper bound where the constant is one up to some additional terms that guarantee reliability. The lower bound is also established. Moreover these additional terms are negligible when the recovered gradient is superconvergent. The reliability and efficiency of the proposed estimator is confirmed by some numerical tests.
\end{abstract}

Key Words A posteriori estimator, Discontinuous Galerkin finite elements. AMS (MOS) subject classification 65N30; 65N15, 65N50,

\section{Introduction}

Among other methods, the finite element method is one of the more popular that is commonly used in the numerical realization of different problems appearing in engineering applications, like the Laplace equation, the Lamé system, the Stokes system, the Maxwell system, etc.... (see $[7,8,24])$. More recently discontinuous Galerkin finite element methods become very attractive since they present some advantages. For example, they allow to do some " $p$ refinement", by locally increasing the polynomial degree of the approximation if needed. They can moreover use non-conform meshes allowing hanging-nodes, making the mesh generation easier for concrete industrial cases. In our days a vast literature exists on

\footnotetext{
*Université des Sciences et Technologies de Lille, Laboratoire Paul Painlevé UMR 8524, EPI SIMPAF INRIA Lille Nord Europe, UFR de Mathématiques Pures et Appliquées, Cité Scientifique, 59655 Villeneuve d'Ascq Cedex email: creuse@math.univ-lille1.fr

${ }^{\dagger}$ Université de Valenciennes et du Hainaut Cambrésis, LAMAV, FR CNRS 2956, Institut des Sciences et Techniques de Valenciennes, F-59313 - Valenciennes Cedex 9 France, email: Serge.Nicaise@univvalenciennes.fr
} 
the subject, we refer to $[3,10]$ and the references cited there. Adaptive techniques based on a posteriori error estimators have become indispensable tools for such methods. For continuous Galerkin finite element methods, there now exists a vast amount of literature on a posteriori error estimation for problems in mechanics or electromagnetism and obtaining locally defined a posteriori error estimates. We refer to the monographs [2, 4, 25, 33] for a good overview on this topic. On the other hand a similar theory for discontinuous methods is less developed, let us quote [5, 12, 17, 18, 20, 28, 31].

Usually upper and lower bounds are proved in order to guarantee the reliability and the efficiency of the proposed estimator. Most of the existing approaches involve constants depending on the shape regularity of the elements and/or of the jumps in the coefficients; but these dependences are often not given. Only a small number of approaches gives rise to estimates with explicit constants, let us quote $[2,6,21,23,26,27,16]$ for continuous methods. For discontinuous methods, we may cite the recent papers $[1,22,9,14,15]$.

Our goal is therefore to consider second order elliptic operators with discontinuous diffusion coefficients in two-dimensional domains with mixed boundary conditions and a discontinuous Galerkin method with polynomials of any degree. Inspired from the paper [16], which treats the case of continuous diffusion coefficients approximated by a continuous Galerkin method, we further derive an a posteriori estimator with an explicit constant in the upper bound (more precisely 1) up to some additional terms that are usually superconvergent and some oscillating terms. The approach, called gradient recovery by averaging [16] is based on the construction of a Zienkiewicz/Zhu estimator, namely the difference in an appropriate norm of $a \nabla_{h} u_{h}-G u_{h}$, where $\nabla_{h} u_{h}$ is the broken gradient of $u_{h}$ and $G u_{h}$ is a $H$ (div )-conforming approximation of this variable. Here special attention has to be paid due to the assumption that a may be discontinuous. Moreover the non conforming part of the error is managed using a Helmholtz decomposition of the error and a standard Oswald interpolation operator $[20,1]$. Furthermore using standard inverse inequalities, we show that our estimator is locally efficient. Two interests of this approach are first the simplicity of the construction of $G u_{h}$, and secondly its superconvergence property (validated by numerical tests).

The schedule of the paper is as follows: We recall in section 2 the diffusion problem, its numerical approximation and an appropriate Helmholtz decomposition of the error. Section 3 is devoted to the introduction of the estimator based on gradient averaging and the proofs of the upper and lower bounds. The upper bound directly follows from the construction of the estimator and some results from [16], while the lower bound requires the use of some inverse inequalities and a special construction of $G u_{h}$. Finally in section 4 some numerical tests are presented that confirm the reliability and efficiency of our estimator and the superconvergence of $G u_{h}$ to $a \nabla u$.

Let us finish this introduction with some notation used in the remainder of the paper: On $D$, the $L^{2}(D)$-norm will be denoted by $\|\cdot\|_{D}$. In the case $D=\Omega$, we will drop the index $\Omega$. The usual norm and semi-norm of $H^{s}(D)(s \geq 0)$ are denoted by $\|\cdot\|_{s, D}$ and $|\cdot|_{s, D}$, respectively. Finally, the notation $a \lesssim b$ and $a \sim b$ means the existence of positive constants $C_{1}$ and $C_{2}$, which are independent of the mesh size and of the quantities $a$ and $b$ under consideration such that $a \lesssim C_{2} b$ and $C_{1} b \lesssim a \lesssim C_{2} b$, respectively. In other words, 
the constants may depend on the aspect ratio of the mesh, the diffusion matrix, as well as the polynomial degree $l$ (see below).

\section{The boundary value problem and its discretization}

Let $\Omega$ be a bounded open domain of $\mathbb{R}^{2}$ with a Lipschitz boundary $\Gamma$ that we suppose to be polygonal. We further assume that $\Omega$ is simply connected and that $\Gamma$ is connected. We consider the following elliptic second order boundary value problem with non homogeneous mixed boundary conditions:

$$
\left\{\begin{aligned}
-\operatorname{div}(a \nabla u) & =f \text { in } \Omega, \\
u & =g_{D} \text { on } \Gamma_{D}, \\
a \nabla u \cdot n & =g_{N} \text { on } \Gamma_{N},
\end{aligned}\right.
$$

where $\Gamma=\bar{\Gamma}_{D} \cup \bar{\Gamma}_{N}$ and $\Gamma_{D} \cap \Gamma_{N}=\emptyset$. For convenience we suppose that meas $\Gamma_{D}>0$.

In the sequel, we suppose that $a$ is a symmetric positive definite matrix which is piecewise constant, namely we assume that there exists a partition $\mathcal{P}$ of $\Omega$ into a finite set of Lipschitz polygonal domains $\Omega_{1}, \cdots, \Omega_{J}$ such that, on each $\Omega_{j}, a=a_{j}$ where $a_{j}$ is a symmetric positive definite matrix. The variational formulation of (1) involves the bilinear form

$$
B(u, v)=\int_{\Omega} a \nabla u \cdot \nabla v
$$

and the Hilbert space

$$
H_{D}^{1}(\Omega)=\left\{u \in H^{1}(\Omega): u=0 \text { on } \Gamma_{D}\right\}
$$

Given $f \in L^{2}(\Omega), g_{D} \in H^{\frac{1}{2}}\left(\Gamma_{D}\right)$ and $g_{N} \in L^{2}\left(\Gamma_{N}\right)$, the weak formulation consists in finding $u \in w+H_{D}^{1}(\Omega)$ such that

$$
B(u, v)=\int_{\Omega} f v+\int_{\Gamma_{N}} g_{N} v, \forall v \in H_{D}^{1}(\Omega),
$$

where $w \in H^{1}(\Omega)$ is a lifting for $g_{D}$, i.e., $w=g_{D}$ on $\Gamma_{D}$. Invoking the positiveness of $a$, the bilinear form $B$ is coercive on $H_{D}^{1}(\Omega)$ with respect to the norm $\left(\int_{\Omega}\left|a^{1 / 2} \nabla u\right|^{2}\right)^{1 / 2}$ and this coerciveness guarantees that problem (2) has a unique solution by the Lax-Milgram lemma.

\subsection{Discontinuous Galerkin approximated problem}

Following [20, 3], we consider the following discontinuous Galerkin approximation of our continuous problem: We consider a triangulation $\mathcal{T}$ of $\Omega$, that is a "partition" of $\Omega$ made of triangles $T$ (closed subsets of $\bar{\Omega}$ ) whose edges are denoted by $e$. We assume that this 
triangulation is regular, i.e., for any element $T$, the ratio $\frac{h_{T}}{\rho_{T}}$ is bounded by a constant $\sigma>0$ independent of $T$ and of the mesh size $h=\max _{T \in \mathcal{T}} h_{T}$, where $h_{T}$ is the diameter of $T$ and $\rho_{T}$ the diameter of its largest inscribed ball. We further assume that $\mathcal{T}$ is conforming with the partition $\mathcal{P}$ of $\Omega$, i.e., the matrix $a$ being constant on each $T \in \mathcal{T}$, we then denote by $a_{T}$ the value of $a$ restricted to an element $T$. With each edge $e$ of the triangulation, we denote by $h_{e}$ its length, we associate a unit normal vector $n_{e}$ (whose orientation can be arbitrary chosen) and the so-called patch $\omega_{e}=\cup_{e \in T} T$, the union of triangles having $e$ as edge. For a triangle $T, n_{T}$ stands for the outer unit normal vector of $T$. $\mathcal{E}$ (resp. $\mathcal{N}$ ) represents the set of edges (resp. vertices) of the triangulation. In the sequel, we need to distinguish between edges included into $\Omega, \Gamma_{D}$ or $\Gamma_{N}$, in other words, we set

$$
\begin{aligned}
\mathcal{E}_{\text {int }} & =\{e \in \mathcal{E}: e \subset \Omega\}, \\
\mathcal{E}_{D} & =\left\{e \in \mathcal{E}: e \subset \Gamma_{D}\right\}, \\
\mathcal{E}_{N} & =\left\{e \in \mathcal{E}: e \subset \Gamma_{N}\right\} .
\end{aligned}
$$

For shortness, we also write $\mathcal{E}_{I D}=\mathcal{E}_{\text {int }} \cup \mathcal{E}_{D}$.

Problem (2) is approximated by the (discontinuous) finite element space:

$$
X_{h}=\left\{v_{h} \in L^{2}(\Omega) \mid v_{h \mid T} \in \mathbb{P}_{\ell}(T), T \in \mathcal{T}\right\},
$$

where $\ell$ is a fixed positive integer.

For our further analysis we need to define some jumps and means of elements of $X_{h}$ through any $e \in \mathcal{E}$ of the triangulation. For $e \in \mathcal{E}$ such that $e \subset \Omega$, we denote by $T^{+}$and $T^{-}$the two elements of $\mathcal{T}$ containing $e$. Let $q \in X_{h}$, we denote by $q^{ \pm}$, the traces of $q$ taken from $T^{ \pm}$, respectively. Then we define the mean of $q$ on $e$ by

$$
\{\{q\}\}=\frac{q^{+}+q^{-}}{2}
$$

For $v \in\left[X_{h}\right]^{d}$, we denote similarly

$$
\{\{v\}\}=\frac{v^{+}+v^{-}}{2}
$$

The jump of $q$ on $e$ is now defined as follows:

$$
\llbracket q \rrbracket=q^{+} n_{T^{+}}+q^{-} n_{T^{-}} .
$$

Remark that the jump $\llbracket q \rrbracket$ of $q$ is vector-valued.

For a boundary edge $e$, i. e., $e \subset \partial \Omega$, there exists a unique element $T^{+} \in \mathcal{T}$ such that $e \subset \partial T^{+}$. Therefore the mean and jump of $q$ are defined by $\{\{q\}\}=q^{+}$and $\llbracket q \rrbracket=q^{+} n_{T^{+}}$.

For $q \in X_{h}$, we define its broken gradient $\nabla_{h} q$ in $\Omega$ by :

$$
\left(\nabla_{h} q\right)_{\mid T}=\nabla q_{\mid T}, \forall T \in \mathcal{T}
$$


The space $X_{h}$ is equipped with the norm

$$
\|q\|_{D G, h}:=\left(\sum_{T \in \mathcal{T}}\left\|a^{1 / 2} \nabla q\right\|_{T}^{2}\right)^{1 / 2}+\left(\sum_{e \in \mathcal{E}_{I D}} h_{e}^{-1}\|\llbracket q \rrbracket\|_{e}^{2}\right)^{1 / 2} .
$$

Later on we also need the continuous counterpart of $X_{h}$, namely we introduce

$$
S_{h}=\left\{v_{h} \in C(\bar{\Omega}) \mid v_{h \mid T} \in \mathbb{P}_{\ell}(T), T \in \mathcal{T}\right\},
$$

as well as

$$
S_{h, 1}=\left\{v_{h} \in C(\bar{\Omega}) \mid v_{h \mid T} \in \mathbb{P}_{1}(T), T \in \mathcal{T}\right\}
$$

We further need

$$
X_{h, 1}=\left\{v_{h} \in L^{2}(\Omega) \mid v_{h \mid T} \in \mathbb{P}_{1}(T), T \in \mathcal{T}\right\} .
$$

With these notations, we define the bilinear form $B_{h}(.,$.$) as follows:$

$$
\begin{aligned}
B_{h}\left(u_{h}, v_{h}\right) & :=\sum_{T \in \mathcal{T}} \int_{T} a \nabla u_{h} \cdot \nabla v_{h}-\sum_{e \in \mathcal{E}_{I D}} \int_{e}\left(\left\{\left\{a \nabla_{h} v_{h}\right\}\right\} \cdot \llbracket u_{h} \rrbracket+\left\{\left\{a \nabla_{h} u_{h}\right\}\right\} \cdot \llbracket v_{h} \rrbracket\right) \\
& +\sum_{e \in \mathcal{E}_{I D}} \gamma_{e} h_{e}^{-1} \int_{e} \llbracket u_{h} \rrbracket \cdot \llbracket v_{h} \rrbracket, \quad \forall u_{h}, v_{h} \in X_{h},
\end{aligned}
$$

where the positive parameters $\gamma_{e}$ are chosen large enough to ensure coerciveness of the bilinear form $B_{h}$ on $X_{h}$ (see, e.g., Lemma 2.1 of [20]), namely according to Theorem 3 of [34] (and the arguments from section 3 of [30]), the following choices yield the coerciveness of $B_{h}$ :

$$
\gamma_{e}>c(\ell+1)(\ell+2) \max _{T \subset \omega_{e}} C_{T},
$$

where $C_{T}$ is the largest eigenvalue of the diffusion matrix $a_{T}$ and $c$ is the positive constant such that

$$
h_{e}^{2} \leq c|T|, \forall T \subset \omega_{e},
$$

that exists due to the regularity assumption on the mesh.

The discontinuous Galerkin approximation of problem (2) reads now: Find $u_{h} \in X_{h}$, such that

$$
B_{h}\left(u_{h}, v_{h}\right)=F\left(v_{h}\right),
$$

where

$$
F\left(v_{h}\right)=\int_{\Omega} f v_{h}+\sum_{e \in \mathcal{E}_{D}} \int_{e} g_{D}\left(\gamma h_{e}^{-1} v_{h}-a \nabla v_{h} \cdot n_{T}\right)+\int_{\Gamma_{N}} g_{N} v_{h}, \forall v_{h} \in X_{h} .
$$

As our approximated scheme is a non conforming one (i.e. the solution does not belong to $H_{D}^{1}(\Omega)$ ), as usual we need to use an appropriate Helmholtz decomposition of the error (see Lemma 3.2 of [13] or Theorem 1 of [1] in 2D). 
Lemma 2.1 (Helmholtz decomposition of the error) We have the following error decomposition

$$
a \nabla_{h}\left(u-u_{h}\right)=a \nabla \varphi+\operatorname{curl} \chi
$$

with $\chi \in H^{1}(\Omega)$ such that

$$
\operatorname{curl} \chi \cdot n=0 \text { on } \Gamma_{N} \text {, }
$$

and $\varphi \in H_{D}^{1}(\Omega)$. Moreover the next identity holds:

$$
\left\|a^{1 / 2} \nabla_{h}\left(u-u_{h}\right)\right\|^{2}=\left\|a^{1 / 2} \nabla_{h} \varphi\right\|^{2}+\left\|a^{-1 / 2} \operatorname{curl} \chi\right\|^{2} .
$$

For the detailed proof of this Lemma we refer to Lemma 2.1 of [9].

\section{The a posteriori error analysis based on gradient recovery by averaging}

Error estimators can be constructed in many different ways as, for example, using residual type error estimators which measure locally the jump of the discrete flux [20]. A different method, based on equilibrated fluxes, consists in solving local Neumann boundary value problems [2] or in using Raviart-Thomas interpolant [1, 9, 14, 15]. Here, as an alternative we introduce a gradient recovery by averaging and define an error estimator based on a $H$ (div)-conforming approximation of this variable. In comparison with [16], we here allow the case of discontinuous diffusion coefficient and use a discontinuous Galerkin method.

Inspired from [16] the conforming part of the estimator $\eta_{C F}$ involves the difference between the broken gradient $a \nabla_{h} u_{h}$ and its smoothed version $G u_{h}$, where $G u_{h}$ is for the moment any element in $X_{h, 1}^{2}$ satisfying

$$
\begin{aligned}
& G u_{h} \in H(\operatorname{div}, \Omega)=\left\{v \in L^{2}(\Omega)^{2}: \operatorname{div} v \in L^{2}(\Omega)\right\} \\
& \left.\left(G u_{h}\right)\right|_{\Omega_{j}} \in H^{1}\left(\Omega_{j}\right), \forall j=1, \cdots, J .
\end{aligned}
$$

Hence conforming part of the estimator $\eta_{C F}$ is defined by

$$
\eta_{C F}^{2}=\sum_{T \in \mathcal{T}} \eta_{C F, T}^{2}
$$

where the indicator $\eta_{C F, T}$ is defined by

$$
\eta_{C F, T}=\left\|a^{-1 / 2}\left(a \nabla u_{h}-G u_{h}\right)\right\|_{T} .
$$

For the nonconforming part of the error, we associate with $u_{h}$, its Oswald interpolation operator, namely the unique element $w_{h} \in S_{h}$ defined in the following natural way (see Theorem 2.2 of [20]): to each node $n$ of the mesh corresponding to the Lagrangian-type degrees of freedom of $S_{h}$, the value of $w_{h}$ is the average of the values of $u_{h}$ at this node $n$ if it belongs to $\Omega \cup \Gamma_{N}$ (i.e., $w_{h}(n)=\frac{\sum_{n \in T}|T| u_{h \mid T}(n)}{\sum_{n \in T}|T|}$ ) and the value of $g_{D}$ at this node if 
it belongs to $\bar{\Gamma}_{D}$ (here we assume that $g_{D} \in C\left(\bar{\Gamma}_{D}\right)$ ). Then the non conforming indicator $\eta_{N C, T}$ is simply

$$
\eta_{N C, T}=\left\|a^{1 / 2} \nabla\left(w_{h}-u_{h}\right)\right\|_{T} .
$$

The non conforming part of the estimator is then

$$
\eta_{N C}^{2}=\sum_{T \in \mathcal{T}} \eta_{N C, T}^{2}
$$

Similarly we introduce the estimator corresponding to jumps of $u_{h}$ :

$$
\eta_{J}^{2}=\sum_{e \in \mathcal{E}_{I D}} \eta_{J, e}^{2}
$$

with

$$
\eta_{J, e}^{2}= \begin{cases}\frac{1}{h_{e}}\left\|\llbracket u_{h} \rrbracket\right\|_{e}^{2} & \text { if } e \in \mathcal{E}_{i n t}, \\ \frac{1}{h_{e}}\left\|u_{h}-g_{D}\right\|_{e}^{2} & \text { if } e \in \mathcal{E}_{D} .\end{cases}
$$

As in [16], we introduce some additional superconvergent security parts. In order to define them properly we recall that for a node $x \in \mathcal{N}$, we denote by $\lambda_{x}$ the standard hat function (defined as the unique element in $S_{h, 1}$ such that $\lambda_{x}(y)=\delta_{x, y}$ for all $y \in \mathcal{N}$ ), let $\omega_{x}$ be the patch associated with $x$, which is simply the support of $\lambda_{x}$ and let $h_{x}$ be the diameter of $\omega_{x}$ (which is equivalent to the diameter $h_{K}$ of any triangle $K$ included into $\left.\omega_{x}\right)$. We now denote by $r$ the element residual

$$
r=f+\operatorname{div}\left(G u_{h}\right)
$$

and for all $x \in \mathcal{N}$, we set

$$
\begin{aligned}
& \bar{r}_{x}=\left(\int_{\omega_{x}} \lambda_{x}\right)^{-1} \int_{\omega_{x}} r \lambda_{x} \quad \text { if } x \in \mathcal{N} \backslash \mathcal{N}_{D} \text {, } \\
& \bar{r}_{x}=\quad 0 \quad \text { if } x \in \mathcal{N}_{D} .
\end{aligned}
$$

We further use a multilevel decomposition of $S_{h, 1}$, namely we suppose that we start from a coarse grid $\mathcal{T}_{0}$ and that the successive triangulations are obtained by using the bisection method, see $[29,16]$. This means that we obtain a finite sequence of nested triangulations $\mathcal{T}_{\ell}, \ell=0, \cdots, L$ such that $\mathcal{T}_{L}=\mathcal{T}$. Denoting by $S_{\ell}$ the space

$$
S_{\ell}=\left\{v \in C(\bar{\Omega}) \mid v_{\mid T} \in \mathbb{P}_{1}(T), T \in \mathcal{T}_{\ell}\right\}
$$

then we have

$$
S_{\ell} \subset S_{\ell+1} \text { and } S_{h, 1}=\cup_{\ell=0}^{L} S_{\ell}=S_{L} .
$$

Furthermore if we denote by $\mathcal{N}_{\ell}$ the nodes of the triangulation $\mathcal{T}_{\ell}$, we have

$$
\mathcal{N}_{\ell} \subset \mathcal{N}_{\ell+1}
$$


As usual for all $z \in \mathcal{N}_{\ell}$ we denote by $\lambda_{\ell z}$ the hat function associated with $z$, namely the unique element in $S_{\ell}$ such that

$$
\lambda_{\ell z}\left(z^{\prime}\right)=\delta_{z z^{\prime}} \forall z^{\prime} \in \mathcal{N}_{\ell} .
$$

For all $\ell \geq 1$ we finally set

$$
\tilde{\mathcal{N}}_{\ell}=\left(\mathcal{N}_{\ell} \backslash \mathcal{N}_{\ell-1}\right) \cup\left\{z \in \mathcal{N}_{\ell-1}: \lambda_{\ell z} \neq \lambda_{\ell-1 z}\right\},
$$

and $\tilde{\mathcal{N}}_{0}=\mathcal{N}_{0}$. It should be noticed (see for instance [16]) that to each $z \in \tilde{\mathcal{N}}_{\ell}$, the corresponding hat function $\lambda_{\ell z}$ does not belong to $S_{\ell-1}$.

Now we define $\bar{\rho}$ and $\bar{\gamma}$ by

$$
\begin{aligned}
\bar{\rho}^{2} & =\sum_{x \in \mathcal{N}} \rho_{x}^{2}, \\
\bar{\gamma}^{2} & =\sum_{\ell=0}^{L} \sum_{z \in \tilde{\mathcal{N}}_{\ell} \backslash \Gamma_{D}} \gamma_{\ell z}^{2},
\end{aligned}
$$

where

$$
\begin{aligned}
\rho_{x}^{2} & =h_{x}^{2} \int_{\omega_{x}}\left|r-\bar{r}_{x}\right|^{2} \lambda_{x}+h_{x} \int_{\omega_{x} \cap \Gamma_{N}}\left|G u_{h} \cdot n-g_{N}\right|^{2} \lambda_{x}, \\
\gamma_{\ell z} & =\left|\left\langle R, \lambda_{\ell z}\right\rangle\right|,
\end{aligned}
$$

$R$ being the residual defined by

$$
\langle R, \varphi\rangle=\int_{\Omega} G u_{h} \cdot \nabla \varphi-\int_{\Omega} f \varphi-\int_{\Gamma_{N}} g_{N} \varphi, \forall \varphi \in H^{1}(\Omega) .
$$

\subsection{Upper bound}

Theorem 3.1 Assume that there exists $w_{h} \in X_{h} \cap H^{1}(\Omega)$ such that $g_{D}=w_{h \mid \Gamma_{D}}$. Let $u \in w+H_{D}^{1}(\Omega)$ be a solution of problem (2) and let $u_{h}$ be its discontinuous Galerkin approximation, i.e. $u_{h} \in X_{h}$ solution of (5). Then there exists $C>0$ such that

$$
\left\|a^{1 / 2} \nabla_{h}\left(u-u_{h}\right)\right\| \leq\left(\eta_{C F}^{2}+\eta_{N C}^{2}\right)^{1 / 2}+C(\bar{\rho}+\bar{\gamma})
$$

and consequently

$$
\left\|u-u_{h}\right\|_{D G, h} \leq\left(\eta_{C F}^{2}+\eta_{N C}^{2}\right)^{1 / 2}+\eta_{J}+C(\bar{\rho}+\bar{\gamma}) .
$$

Remark 3.2 Let us note that under a superconvergence property of $\left\|a^{-1 / 2}\left(G u_{h}-a \nabla u\right)\right\|$, $\bar{\rho}$ and $\bar{\gamma}$ will be proved to be negligible quantities (see Theorem 3.5 below), so that the error is in this case asymptotically bounded above by the estimator without any multiplicative constant. This superconvergence property is observed in most of practical cases, as for example in our numerical tests (see section 4). Moreover, theoretical results for different continuous finite element methods on structured and unstructured meshes have been established (see for example [19, 32, 35]), but, to our knowledge, not yet for discontinuous methods on unstructured multi-dimensional meshes. 
Proof: From the Helmholtz decomposition of the error we have

$$
\left\|a^{1 / 2} \nabla_{h}\left(u-u_{h}\right)\right\|^{2}=\left\|a^{1 / 2} \nabla \varphi\right\|^{2}+\left\|a^{-1 / 2} \operatorname{curl} \chi\right\|^{2} .
$$

We are then reduced to estimate each term of this right-hand side.

For the non conforming part, we proceed as in [1], namely by Green's formula we have

$$
\begin{aligned}
\left\|a^{-1 / 2} \operatorname{curl} \chi\right\|^{2} & =\int_{\Omega} \nabla_{h}\left(u-u_{h}\right) \cdot \operatorname{curl} \chi \\
& =-\int_{\Omega} \nabla_{h} u_{h} \cdot \operatorname{curl} \chi+\int_{\Gamma_{D}} g_{D} \operatorname{curl} \chi \cdot n \\
& =\int_{\Omega} \nabla_{h}\left(w_{h}-u_{h}\right) \cdot \operatorname{curl} \chi,
\end{aligned}
$$

since $\int_{\Omega} \nabla w_{h} \cdot \operatorname{curl} \chi=\int_{\Gamma_{D}} g_{D} \operatorname{curl} \chi \cdot n$. By Cauchy-Schwarz's inequality we directly obtain

$$
\left\|a^{-1 / 2} \operatorname{curl} \chi\right\|^{2} \leq \eta_{N C}\left\|a^{-1 / 2} \operatorname{curl} \chi\right\| .
$$

For the conforming part, we write

$$
\begin{aligned}
\left\|a^{1 / 2} \nabla \varphi\right\|^{2} & =\int_{\Omega} a \nabla_{h}\left(u-u_{h}\right) \cdot \nabla \varphi \\
& =\int_{\Omega}\left(a \nabla u-G u_{h}\right) \cdot \nabla \varphi+\int_{\Omega}\left(G u_{h}-a \nabla_{h} u_{h}\right) \cdot \nabla \varphi .
\end{aligned}
$$

By Cauchy-Schwarz's inequality we obtain

$$
\left\|a^{1 / 2} \nabla \varphi\right\|^{2} \leq\left\|a^{-1 / 2}\left(a \nabla_{h} u_{h}-G u_{h}\right)\right\|\left\|a^{1 / 2} \nabla \varphi\right\|+\left|\int_{\Omega}\left(a \nabla u-G u_{h}\right) \cdot \nabla \varphi\right| .
$$

Using problem (2), the second term of this right-hand side is bounded by the residual, indeed

$$
-\int_{\Omega}\left(a \nabla u-G u_{h}\right) \cdot \nabla \varphi=\int_{\Omega} G u_{h} \cdot \nabla \varphi-\int_{\Omega} f \varphi-\int_{\Gamma_{N}} g_{N} \varphi=\langle R, \varphi\rangle .
$$

Using the arguments from Theorem 4.1 of [16], we have

$$
|\langle R, \varphi\rangle| \leq C(\bar{\rho}+\bar{\gamma})\left\|a^{1 / 2} \nabla \varphi\right\| .
$$

Coming back to the identity (15), and using the estimates (16), (17) and (18) we conclude by discrete Cauchy-Schwarz's inequality and again using (15):

$$
\begin{aligned}
\left\|a^{1 / 2} \nabla_{h}\left(u-u_{h}\right)\right\|^{2} & \leq \eta_{N C}\left\|a^{-1 / 2} \operatorname{curl} \chi\right\|+\left(\eta_{C F}+C(\bar{\rho}+\bar{\gamma})\right)\left\|a^{1 / 2} \nabla \varphi\right\| \\
& \leq\left(\eta_{N C}^{2}+\eta_{C F}^{2}\right)^{1 / 2}\left(\left\|a^{-1 / 2} \operatorname{curl} \chi\right\|^{2}+\left\|a^{1 / 2} \nabla \varphi\right\|^{2}\right)^{1 / 2} \\
& +C(\bar{\rho}+\bar{\gamma})\left\|a^{1 / 2} \nabla \varphi\right\| \\
& \leq\left[\left(\eta_{N C}^{2}+\eta_{C F}^{2}\right)^{1 / 2}+C(\bar{\rho}+\bar{\gamma})\right]\left\|a^{1 / 2} \nabla_{h}\left(u-u_{h}\right)\right\| .
\end{aligned}
$$




\subsection{Lower bound}

Our lower bound is based on the equivalence of the local $L^{2}$-norm of any element in $X_{h}$ with a local $L^{2}$-norm in the interfaces. First of all for any vertex $x$ of one $\Omega_{j}$ and belonging to more than one sub-domain, we introduce the following local notation: let $\Omega_{i}, i=1, \cdots, n$, $n \geq 2$, the sub-domains that have $x$ as vertex. We further denote by $n_{i}$ the unit normal vector along the interface $I_{i}$ between $\Omega_{i}$ and $\Omega_{i+1}$ (modulo $n$ if $x$ is inside the domain $\Omega$ ) and oriented from $\Omega_{i}$ and $\Omega_{i+1}$. Now we are able to prove the following lemma:

Lemma 3.3 Assume that $x$ is a vertex of one $\Omega_{j}$ and belonging to more than one subdomain, and use the notations introduced above. Then there exists a positive constant $C$ that depends only on the geometrical situation of the $\Omega_{i}$ 's near $x$ such that for all $v^{(i)} \in \mathbb{R}^{2}$, $i=1, \cdots, n$, there exist vectors $g(v)^{(i)} \in \mathbb{R}^{2}, i=1, \cdots, n$ satisfying

$$
\left(g(v)^{(i+1)}-g(v)^{(i)}\right) \cdot n_{i}=0, \forall i=1, \cdots, n,
$$

and such that the following estimate holds

$$
\sum_{i=1}^{n}\left|v^{(i)}-g(v)^{(i)}\right| \leq C \sum_{i=1}^{n}\left|\llbracket v \cdot n \rrbracket_{i}\right|,
$$

where here $|\cdot|$ means the Euclidean norm and $\llbracket v \cdot n \rrbracket_{i}$ means the normal jump of $v$ along the interface $I_{i}$ :

$$
\llbracket v \cdot n \rrbracket_{i}=\left(v^{(i+1)}-v^{(i)}\right) \cdot n_{i}, \forall i=1, \cdots, n .
$$

Proof: First introduce the following subspace of $\mathbb{R}^{2 n}$ :

$$
W=\left\{v=\left(v^{(i)}\right)_{i=1}^{n}: v^{(i)} \in \mathbb{R}^{2} \text { and satisfying } \llbracket v \cdot n \rrbracket_{i}=0, \forall i=1, \cdots, n\right\} .
$$

We take $g(v)=\Pi_{W} v$, the orthogonal projection of $v=\left(v^{(i)}\right)_{i=1}^{n}$ into $W$. By construction $g(v)$ trivially satisfies (19). On the other hand the estimate (20) is equivalent to

$$
\left|v-\Pi_{W} v\right| \leq C \sum_{i=1}^{n}\left|\llbracket v \cdot n \rrbracket_{i}\right|,
$$

which is easily proved by a contradiction argument and the fact that we are in a finite dimensional space.

Using the above lemma, we are now able to prove the asymptotic nondeterioration of the smoothed gradient if the following choice for $G u_{h}$ is made: We distinguish the following different possibilities for $x \in \mathcal{N}$.

1) First for all vertex $x$ of the mesh (i.e. vertex of at least one triangle) such that $x$ is inside one $\Omega_{j}$, we set

$$
\left(G u_{h}\right)_{\mid \Omega_{j}}(x)=\frac{1}{\left|\omega_{x}\right|} \sum_{x \in T}|T| a_{T} \nabla u_{h \mid T}(x) .
$$


2) Second if $x$ belongs to the boundary of $\Omega$ and to the boundary of only one $\Omega_{j}$ (hence it does not belong to the boundary of another $\left.\Omega_{k}\right)$, we define $\left(G u_{h}\right)_{\mid \Omega_{j}}(x)$ as before.

3) If $x$ belongs to an interface between two different sub-domain $\Omega_{j}$ and $\Omega_{k}$ but is not a vertex of these sub-domains, then we denote by $n_{j, k}$ the unit normal vector pointing from $\Omega_{j}$ to $\Omega_{k}$ and set $t_{j, k}$ the unit orthogonal vector of $n_{j, k}$ so that $\left(n_{j, k}, t_{j, k}\right)$ is a direct basis of $\mathbb{R}^{2}$; in that case we set

$$
\begin{aligned}
\left(G u_{h}\right)_{\mid \Omega_{j}}(x) \cdot n_{j, k} & =\left(G u_{h}\right)_{\mid \Omega_{k}}(x) \cdot n_{j, k}=\frac{1}{\left|\omega_{x}\right|} \sum_{x \in T}|T| a_{T} \nabla u_{h \mid T}(x) \cdot n_{j, k}, \\
\left(G u_{h}\right)_{\mid \Omega_{j}}(x) \cdot t_{j, k} & =\frac{1}{\left|\omega_{x} \cap \Omega_{j}\right|} \sum_{T \subset \Omega_{j}: x \in T}|T| a_{T} \nabla u_{h \mid T}(x) \cdot t_{j, k}, \\
\left(G u_{h}\right)_{\mid \Omega_{k}}(x) \cdot t_{j, k} & =\frac{1}{\left|\omega_{x} \cap \Omega_{k}\right|} \sum_{T \subset \Omega_{k}: x \in T}|T| a_{T} \nabla u_{h \mid T}(x) \cdot t_{j, k} .
\end{aligned}
$$

4) Finally if $x$ is a vertex of at least two sub-domains $\Omega_{j}$, for the sake of simplicity we suppose that each triangle $T$ having $x$ as vertex is included into one $\Omega_{j}$, and we take

$$
\left(G u_{h}\right)_{\mid \Omega_{j}}(x)=g(v)^{(j)} \forall j \in \mathcal{J}_{x},
$$

where $\mathcal{J}_{x}=\left\{j \in\{1, \cdots, J\}: x \in \bar{\Omega}_{j}\right\}, g(v)^{(j)}$ were defined in the previous Lemma 3.3 with here $v$ given by $v=\left(a_{j} \nabla u_{h \mid T}(x)\right)_{j \in \mathcal{J}_{x}}$.

With these choices, we take

$$
\left(G u_{h}\right)_{\mid \Omega_{j}}=\sum_{x \in \mathcal{N} \cap \bar{\Omega}_{j}}\left(G u_{h}\right)_{\mid \Omega_{j}}(x) \lambda_{x}, \forall j=1, \cdots, J
$$

where $\left(G u_{h}\right)_{\mid \Omega_{j}}(x)$ was defined before.

The main point is that by construction $G u_{h}$ satisfies the requirements (9) and (10) but moreover we have the next asymptotic nondeterioration result:

Theorem 3.4 If $\ell \leq 2$ (see (3)), then for each element $T \in \mathcal{T}$ the following estimate holds

$$
\left\|a_{T}^{-1 / 2}\left(G u_{h}-a_{T} \nabla u\right)\right\|_{T} \lesssim\left\|u-u_{h}\right\|_{D G, \omega_{T}}+\operatorname{osc}\left(f, \omega_{T}\right),
$$

where $\omega_{T}$ denotes the patch consisting of all the triangles of $\mathcal{T}$ having a nonempty intersection with $T$ and

$$
\|v\|_{D G, \omega_{T}}^{2}=\left\|a^{1 / 2} \nabla_{h} v\right\|_{\omega_{T}}^{2}+\gamma \sum_{e \in \mathcal{E}_{I D}: e \subset \omega_{T}} h_{e}^{-1}\|\llbracket v \rrbracket\|_{e}^{2},
$$

and

$$
\operatorname{osc}\left(f, \omega_{T}\right)^{2}=h_{T}^{2} \sum_{T^{\prime} \subset \omega_{T}}\left\|f-\Pi_{T^{\prime}} f\right\|_{T^{\prime}}^{2},
$$

where $\Pi_{T^{\prime}} f$ is the $L^{2}\left(T^{\prime}\right)$-orthogonal projection of $f$ onto $\mathbb{P}_{1}\left(T^{\prime}\right)$. 
Proof: By the triangle inequality we may write

$$
\begin{array}{r}
\left\|a_{T}^{-1 / 2}\left(G u_{h}-a_{T} \nabla u\right)\right\|_{T} \leq\left\|a_{T}^{-1 / 2}\left(G u_{h}-a_{T} \nabla u_{h}\right)\right\|_{T}+\left\|a_{T}^{-1 / 2}\left(a_{T} \nabla u_{h}-a_{T} \nabla u\right)\right\|_{T} \\
\leq\left\|a_{T}^{-1 / 2}\left(G u_{h}-a_{T} \nabla u_{h}\right)\right\|_{T}+\left\|u-u_{h}\right\|_{D G, T} .
\end{array}
$$

Therefore it remains to estimate the first term of this right-hand side. For that purpose, since $T \subset \Omega_{j}$ for a unique $j \in\{1, \cdots, J\}$, we may write

$$
\left(G u_{h}-a_{T} \nabla u_{h}\right)_{\mid T}=\sum_{x \in T}\left\{\left(G u_{h}\right)_{\mid \Omega_{j}}(x)-a_{j} \nabla u_{h \mid T}(x)\right\} \lambda_{x} .
$$

As $0 \leq \lambda_{x} \leq 1$, and since the triangulation is regular, we get

$$
\left\|a_{T}^{-1 / 2}\left(G u_{h}-a_{T} \nabla u_{h}\right)\right\|_{T} \lesssim \sum_{x \in T}\left|\left(G u_{h}\right)_{\mid \Omega_{j}}(x)-a_{j} \nabla u_{h \mid T}(x)\right| h_{T} .
$$

We are then reduced to estimate the factor $\left|\left(G u_{h}\right)_{\mid \Omega_{j}}(x)-a_{j} \nabla u_{h \mid T}(x)\right|$ for all nodes $x$ of $T$. For that purpose, we distinguish four different cases:

1) If $x \in \Omega_{j}$, then we use an argument similar to the one from Proposition 4.2 of [16] adapted to the DG situation. By the definition of $G u_{h}$, we have

$$
\left(G u_{h}\right)_{\mid \Omega_{j}}(x)=\frac{1}{\left|\omega_{x}\right|} \sum_{T^{\prime} \subset \omega_{x}}\left|T^{\prime}\right| a_{j} \nabla u_{h \mid T^{\prime}}(x),
$$

because in this case all $T^{\prime} \subset \omega_{x}$ are included into $\Omega_{j}$. As a consequence, we obtain

$$
\left(G u_{h}\right)_{\mid \Omega_{j}}(x)-a_{j} \nabla u_{h \mid T}(x)=\frac{1}{\left|\omega_{x}\right|} \sum_{T^{\prime} \subset \omega_{x}}\left|T^{\prime}\right| a_{j}\left(\nabla u_{h \mid T^{\prime}}(x)-\nabla u_{h \mid T}(x)\right),
$$

and therefore

$$
\left|\left(G u_{h}\right)_{\mid \Omega_{j}}(x)-a_{j} \nabla u_{h \mid T}(x)\right| \lesssim \sum_{T^{\prime} \subset \omega_{x}}\left|a_{j}\left(\nabla u_{h \mid T^{\prime}}(x)-\nabla u_{h \mid T}(x)\right)\right| .
$$

For each $T^{\prime} \subset \omega_{x}$, there exists a path of triangles of $\omega_{x}$, written $T_{i}, i=0, \cdots, n$ such that

$$
T_{0}=T, T_{n}=T^{\prime}, T_{i} \neq T_{j}, \forall i \neq j,
$$

$T_{i} \cap T_{i+1}$ is an common edge $\forall i=1, \cdots, n-1$.

Hence by the triangle inequality we can estimate

$$
\left|a_{j}\left(\nabla u_{h \mid T^{\prime}}(x)-\nabla u_{h \mid T}(x)\right)\right| \leq \sum_{i=0}^{n-1}\left|a_{j}\left(\nabla u_{h \mid T_{i+1}}(x)-\nabla u_{h \mid T_{i}}(x)\right)\right| .
$$

Now for each term, since $a_{j}$ is symmetric and positive definite, we have $\left|a_{j}\left(\nabla u_{h \mid T_{i+1}}(x)-\nabla u_{h \mid T_{i}}(x)\right)\right| \lesssim\left|\left\{a_{j}\left(\nabla u_{h \mid T_{i+1}}(x)-\nabla u_{h \mid T_{i}}(x)\right)\right\} \cdot n_{i}\right|+\left|\left(\nabla u_{h \mid T_{i+1}}(x)-\nabla u_{h \mid T_{i}}(x)\right) \cdot t_{i}\right|$, 
where $n_{i}$ is one fixed unit normal vector along the edge $T_{i} \cap T_{i+1}$ and $t_{i}$ is one fixed unit tangent vector along this edge.

All together we have shown that

$$
\left|\left(G u_{h}\right)_{\mid \Omega_{j}}(x)-a_{j} \nabla u_{h \mid T}(x)\right| h_{T} \lesssim h_{T} \sum_{e \in \mathcal{E}_{\text {int }}: e \subset \omega_{x}}\left\{\left|\llbracket a_{j} \nabla u_{h}(x) \cdot n \rrbracket_{e}\right|+\left|\llbracket \nabla-h u_{h}(x) \cdot t \rrbracket_{e}\right|\right\} .
$$

Using a norm equivalence and an inverse inequality we obtain

$$
\left|\left(G u_{h}\right)_{\mid \Omega_{j}}(x)-a_{j} \nabla u_{h \mid T}(x)\right| h_{T} \lesssim \sum_{e \in \mathcal{E}_{\text {int }}: \subset \subset \omega_{x}}\left\{h_{e}^{1 / 2}\left\|\llbracket a_{j} \nabla_{h} u_{h} \cdot n \rrbracket_{e}\right\|_{e}+h_{e}^{-1 / 2}\left\|\llbracket u_{h} \rrbracket\right\|_{e}\right\} .
$$

2 ) If the node $x$ belongs to the boundary of $\Omega$ and to the boundary of a unique $\Omega_{j}$, since $\left(G u_{h}\right)_{\mid \Omega_{j}}(x)$ is defined as in the first case, the above arguments lead to (29).

3 ) If $x$ is a vertex of different sub-domains $\Omega_{j}$, then by Lemma 3.3, we have

$$
\left|\left(G u_{h}\right)_{\mid \Omega_{j}}(x)-a_{j} \nabla u_{h \mid T}(x)\right| h_{T} \lesssim h_{T} \sum_{e \in \mathcal{E}_{\text {int }}: e \subset \omega_{x}}\left|\llbracket a \nabla_{h} u_{h}(x) \cdot n \rrbracket_{e}\right|,
$$

and therefore as before we conclude that (29) holds.

4) Finally if $x$ belongs to an interface between two subdomains and is not a vertex of them, then it is not difficult to show that (30) holds (due to the regularity of the mesh), and consequently (29) is still valid.

Summarizing the different cases, by (28) and (29), we have

$$
\left\|a_{T}^{-1 / 2}\left(G u_{h}-a_{T} \nabla u_{h}\right)\right\|_{T} \lesssim \sum_{e \in \mathcal{E}_{\text {int }}: \subset \subset \omega_{x}}\left\{h_{e}^{1 / 2}\left\|\llbracket a \nabla_{h} u_{h} \cdot n \rrbracket_{e}\right\|_{e}+h_{e}^{-1 / 2}\left\|\llbracket u_{h} \rrbracket\right\|_{e}\right\} .
$$

The first term of this right hand side is a part of the standard residual error estimator and it is by now standard that (using appropriate bubble functions and Green's formula)

$$
h_{e}^{1 / 2}\left\|\llbracket a \nabla u_{h} \cdot n \rrbracket_{e}\right\|_{e} \lesssim\left\|a \nabla_{h}\left(u-u_{h}\right)\right\|_{\omega_{e}}+\operatorname{osc}\left(f, \omega_{e}\right), \forall e \in \mathcal{E}_{\text {int }} .
$$

The second term is part of the DG-norm. Therefore the above estimate in (31) leads to (27).

Now using the same arguments than in Proposition 4.1 of [16], we have

Theorem 3.5 For all $T \in \mathcal{T}, x \in \mathcal{N}$ and $\ell \geq 0, z \in \mathcal{N}_{\ell}$, we have

$$
\begin{aligned}
\eta_{C F, T} & \leq\left\|a_{T}^{1 / 2} \nabla\left(u_{h}-u\right)\right\|_{T}+\left\|a^{-1 / 2}\left(G u_{h}-a \nabla u\right)\right\|_{T}, \\
\bar{\rho}_{x} & \lesssim\left\|a^{-1 / 2}\left(G u_{h}-a \nabla u\right)\right\|_{\omega_{x}}+\operatorname{osc}\left(f, \omega_{x}\right)+\operatorname{osc}\left(g_{N}, \omega_{x}\right), \\
\bar{\gamma}_{\ell z} & \lesssim\left\|a^{-1 / 2}\left(G u_{h}-a \nabla u\right)\right\|_{\omega_{\ell z}},
\end{aligned}
$$

where

$$
\operatorname{osc}\left(g_{N}, \omega_{x}\right)^{2}=\sum_{e \subset \omega_{x} \cap \Gamma_{N}} h_{e}\left\|g_{N}-\Pi_{e} g_{N}\right\|_{T}^{2}
$$

$\Pi_{e} g_{N}$ being the $L^{2}(e)$-orthogonal projection of $g_{N}$ onto $\mathbb{P}_{2}(e)$. 
For the non conforming part of the estimator, we make use of Theorem 2.2 of [20] to directly obtain the

Theorem 3.6 Let the assumptions of Theorem 3.1 be satisfied. For each element $T \in \mathcal{T}$ the following estimate holds

$$
\eta_{N C, T} \lesssim a_{T}^{1 / 2}\left\|u-u_{h}\right\|_{D G, \omega_{T}}
$$

A direct consequence of these three Theorems is the next local lower bound:

Theorem 3.7 Let the assumptions of Theorems 3.1 and 3.4 be satisfied. For each element $T \in \mathcal{T}$ the following estimate holds

$$
\eta_{C F, T}+\eta_{N C ; T}+\eta_{J, T}+\sum_{x \in T}\left(\bar{\rho}_{x}+\bar{\gamma}_{x}\right) \lesssim\left\|u-u_{h}\right\|_{D G, \omega_{T}}+\operatorname{osc}\left(f, \omega_{T}\right)+\operatorname{osc}\left(g_{N}, \omega_{T}\right),
$$

where $\bar{\gamma}_{x}=\bar{\gamma}_{L x}$ recalling that $L$ is such that $\mathcal{N}_{L}=\mathcal{N}$.

Remark 3.8 Note that the lower bound on the non conforming estimator (see (32)) involves a constant that depends on the aspect ratio of the mesh and of the penalization parameter $\gamma$, and is specific to the discontinuous Galerkin method. Consequently it prevents the estimator to be asymptotically exact, as in the continuous Galerkin method [16]. Nevertheless the numerical tests show quite satisfactory effectivity indices (see below).

As in Proposition 4.3 of [16], one has

$$
\bar{\gamma} \lesssim\left\|a^{-1 / 2}\left(G u_{h}-a \nabla u\right)\right\|
$$

and therefore a global lower bound can be obtained:

Theorem 3.9 Let the assumptions of Theorems 3.1 and 3.4 be satisfied. Then the following global lower bound holds

$$
\eta_{C F}+\eta_{N C}+\eta_{J}+\bar{\rho}+\bar{\gamma} \lesssim\left\|u-u_{h}\right\|_{D G, h}+\operatorname{osc}(f, \Omega)+\operatorname{osc}\left(g_{N}, \Omega\right)
$$

\section{Numerical results}

\subsection{The polynomial solution}

In order to illustrate our theoretical predictions, this first numerical test consists in validating our computations on a simple case, using an uniform refinement process. Let $\Omega$ be the square $(-1,1)^{2}, \Gamma_{D}=\partial \Omega, a=I d$ and $f$ defined such that the exact solution $u$ is given by :

$$
u(x, y)=(x+1)(x-1)(y+1)(y-1) .
$$


Let us recall that $u_{h}$ is the finite element solution, $e\left(u_{h}\right)=\left\|u-u_{h}\right\|_{D G, h}$ the error, $\eta\left(u_{h}\right)=\left(\eta_{C F}^{2}+\eta_{N C}^{2}\right)^{1 / 2}+\eta_{J}$ the estimator and $G u_{h}$ the approximated value of $a \nabla u$ given by (26). We also define $C V_{\text {error }}$ (resp. $C V_{\text {recov }}$ ) as the convergence rate of the error $e\left(u_{h}\right)$ (resp. of the quantity $\left\|G u_{h}-\nabla u\right\|$ ) with respect to $D o F^{-1 / 2}$ from one line of the table to the following one.

Computations are performed using a global mesh refinement process from an initial cartesian grid, using $\gamma_{e} \equiv \gamma=20$ to ensure (4). First, it can be seen from Table 1 that the convergence rate of the numerical method is equal to one, as theoretically expected. Then, the superconvergence property of the term $\left\|G u_{h}-\nabla u\right\|$ is actually observed with a convergence rate of 1.50. Finally, the reliability of the estimator is ensured since the ratio in the last column (the so-called effectivity index), converges fastly towards the constant 1.70.

\begin{tabular}{|c|l|c|c|c|c|c|}
\hline$k$ & $D o F$ & $e\left(u_{h}\right)$ & $C V_{\text {error }}$ & $\left\|G u_{h}-\nabla u\right\|$ & $C V_{\text {recov }}$ & $\frac{\eta\left(u_{h}\right)}{e\left(u_{h}\right)}$ \\
\hline 1 & 384 & $3.80 \mathrm{E}-01$ & & $2.78 \mathrm{E}-01$ & & 1.60 \\
\hline 2 & 1536 & $1.90 \mathrm{E}-01$ & 1.00 & $1.04 \mathrm{E}-01$ & 1.42 & 1.67 \\
\hline 3 & 6144 & $9.51 \mathrm{E}-02$ & 1.00 & $3.73 \mathrm{E}-02$ & 1.47 & 1.70 \\
\hline 4 & 24576 & $4.74 \mathrm{E}-02$ & 1.00 & $1.32 \mathrm{E}-02$ & 1.49 & 1.70 \\
\hline 5 & 98304 & $2.37 \mathrm{E}-02$ & 1.00 & $4.69 \mathrm{E}-03$ & 1.50 & 1.70 \\
\hline 6 & 393216 & $1.19 \mathrm{E}-02$ & 1.00 & $1.66 \mathrm{E}-02$ & 1.50 & 1.70 \\
\hline
\end{tabular}

Table 1: The polynomial solution (uniform refinement).

\subsection{The interior and boundary layer solution}

The following numerical test consists in solving the interior and boundary layer example given in [16]. Let $\Omega$ the square $(-1,1)^{2}, \Gamma_{D}=\partial \Omega, a=I d$ and $f$ defined such that :

$$
u(x, y)=\arctan \left(60\left(x^{2}+y^{2}-1\right)\right)
$$

is the exact solution (see Figure 1). Let us note that the boundary layer crosses the boundary $\partial \Omega$ and that the loading term oscillates across it, what constitutes the difficulty of the computation. This time, an adaptive mesh refinement strategy is used based on the estimator $\eta_{T}=\eta_{C F, T}+\eta_{N C, T}+\eta_{J, T}$ and the marking procedure

$$
\eta_{T}>0.75 \max _{T^{\prime}} \eta_{T^{\prime}}
$$

and a standard refinement procedure with a limitation on the minimal angle. Once again, we choose $\gamma_{e} \equiv \gamma=20$. Several mesh levels are displayed on Figure 2, to show the capability of the algorithm to track the high gradients regions. Furthermore, quantitative 


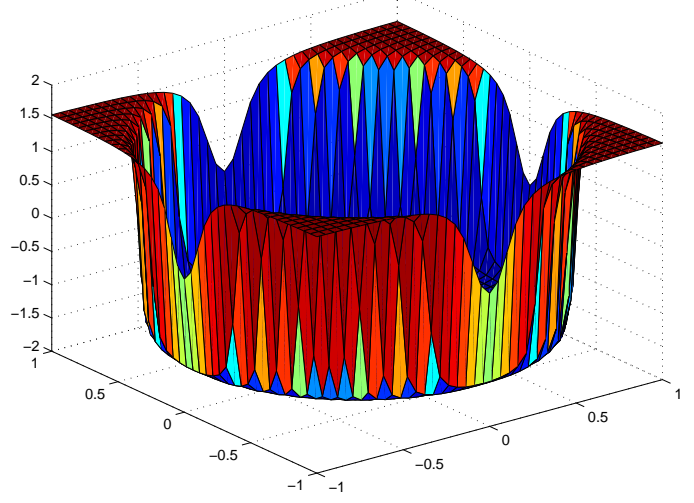

Figure 1: Interior and boundary layer solution.

results are displayed on Table 2 . Once again, the superconvergence property of $\left\|G u_{h}-\nabla u\right\|$ is asymptotically observed, as well as the reliability of the estimator, provided the mesh is fine enough around the boundary layer.
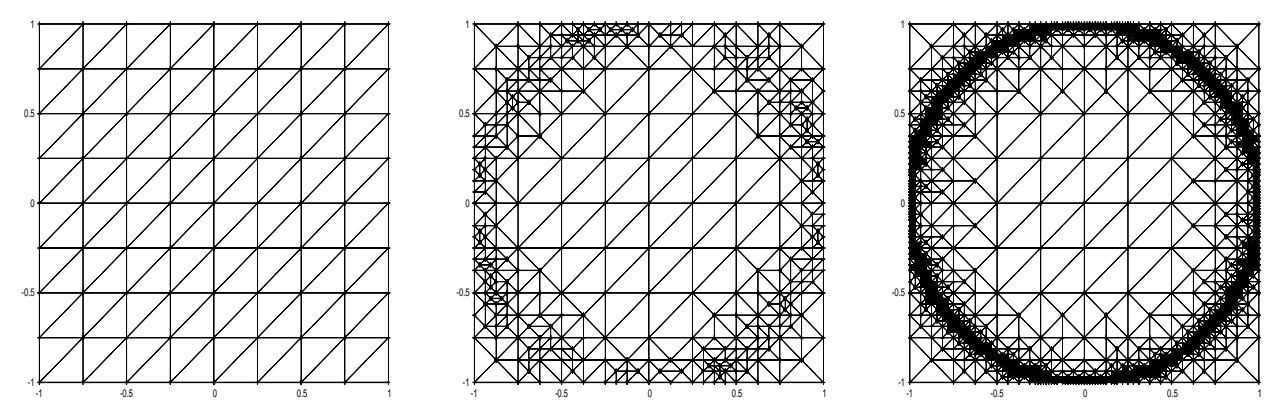

Figure 2: Mesh levels 1, 10 and 20, interior and boundary layer solution.

\subsection{The discontinuous case}

This section is devoted to the case of the discontinuous coefficient $a$. Namely, the domain $\Omega=(-1,1)^{2}$ with $\Gamma_{D}=\Gamma$ is decomposed into 4 sub-domains $\Omega_{i}, i=1, \ldots, 4$, with $\Omega_{1}=$ $(0,1) \times(0,1), \Omega_{2}=(-1,0) \times(0,1), \Omega_{3}=(-1,0) \times(-1,0)$ and $\Omega_{4}=(0,1) \times(-1,0)$. In that case we take discontinuous coefficient $a$, namely we take $a=a_{i}$ on $\Omega_{i}$, with $a_{1}=a_{3}=I d$ and $a_{2}=a_{4}=C I d$ with $C$ to be specified. For this second test, and using usual polar coordinates centered at $(0,0)$, the exact solution is equal to the singular function $u(x, y)=r^{\alpha} \phi(\theta)$, where $\alpha \in(0,1)$ and $\phi$ are chosen such that $u$ is harmonic on each sub-domain $\Omega_{i}, i=1, . .4$, and satisfies the jump conditions :

$$
[u]=0 \text { and }[a \nabla u \cdot n]=0
$$




\begin{tabular}{|l|l|c|c|c|c|c|}
\hline$k$ & DoF & $e\left(u_{h}\right)$ & $C V_{\text {error }}$ & $\left\|G u_{h}-\nabla u\right\|$ & $C V_{\text {recov }}$ & $\frac{\eta\left(u_{h}\right)}{e\left(u_{h}\right)}$ \\
\hline 1 & 384 & $1.02 \mathrm{E}+02$ & & $4.67 \mathrm{E}+01$ & & 1.10 \\
\hline 7 & 1323 & $4.59 \mathrm{E}+01$ & 1.29 & $3.36 \mathrm{E}+01$ & 0.53 & 0.94 \\
\hline 15 & 6069 & $1.71 \mathrm{E}+01$ & 1.29 & $1.67 \mathrm{E}+01$ & 0.91 & 1.35 \\
\hline 22 & 22458 & $7.84 \mathrm{E}+00$ & 1.19 & $6.55 \mathrm{E}+00$ & 1.43 & 1.67 \\
\hline 28 & 94260 & $3.56 \mathrm{E}+00$ & 1.10 & $1.84 \mathrm{E}+00$ & 1.77 & 1.62 \\
\hline 34 & 366180 & $1.80 \mathrm{E}+00$ & 1.01 & $7.14 \mathrm{E}-01$ & 1.40 & 1.60 \\
\hline
\end{tabular}

Table 2: The interior and boundary layer solution (adaptive refinement).

on the interfaces. Non-homogeneous Dirichlet boundary conditions on $\Gamma$ are fixed accordingly. It is easy to see (see for instance [11]) that $\alpha$ is the root of the transcendental equation

$$
\tan \frac{\alpha \pi}{4}=\sqrt{1 / C}
$$

Since $\alpha<1$, this solution has a singular behavior around the point $(0,0)$. For this test, we also compute the standard ZZ smoothed gradient $G^{*} u_{h}$ belonging to $S_{h, 1}^{(2)}=$ $\left\{v_{h} \in C(\bar{\Omega})^{2} \mid v_{h \mid T} \in \mathbb{P}_{1}^{2}(T), T \in \mathcal{T}\right\}$ and characterized by its value at each node of the mesh given by :

$$
\left(G^{*} u_{h}\right)(x)=\frac{1}{\left|\omega_{x}\right|} \sum_{x \in T}|T| a_{T} \nabla u_{h \mid T}(x) .
$$

For the case $C=5$, we choose $\gamma_{e} \equiv \gamma=20$ and figure 3 shows some of the meshes obtained during the local refinement process, which is the same as the one used in section 4.2. Moreover, table 3 displays the corresponding quantitative results. The smoothed gradient $G u_{h}$ is superconvergent, while the effectivity index converges towards 1.75 , which is quite satisfactory and comparable with results from $[9,15]$ as well as those of the previous tests in sections 4.1 and 4.2 .

For the case $C=100$, we choose $\gamma_{e} \equiv \gamma=500$ and figure 4 shows some of the meshes obtained during the local refinement process. These ones are more refined around the singularity than the case $C=5$. Table 4 displays the corresponding quantitative results. Once again, the smoothed gradient $G u_{h}$ is superconvergent, provided the mesh resolution is fine enough. The effectivity index requires more iterations to converge, but is already equal to 2.82 for the finer mesh resolution.

Let us finally note that for both cases, the standard ZZ smoothed gradient $G^{*} u_{h}$ is no more superconvergent towards $a \nabla u$, as in the case $a=I d$. This is not surprising since the statement of Theorem 3.4 is not valid for $G^{*} u_{h}$ if $a \neq I d$. 

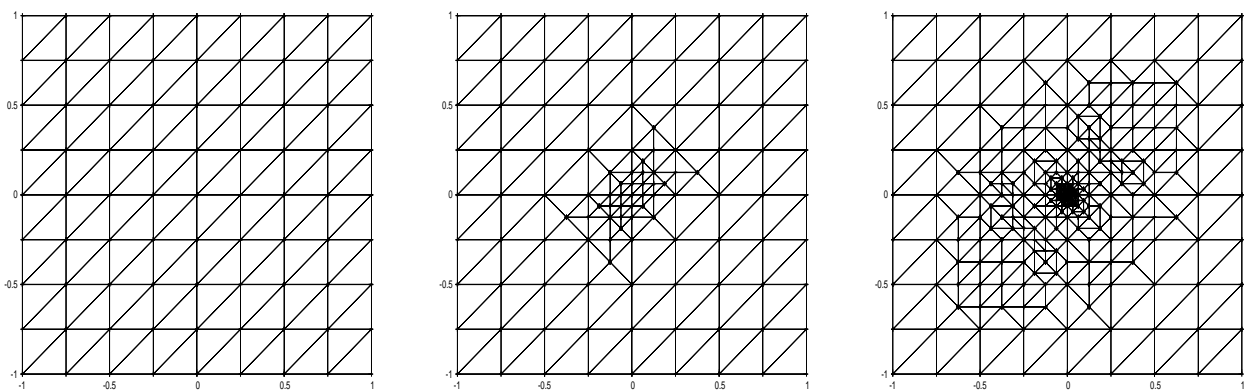

Figure 3: Mesh levels 1, 3 and 10, singular solution for $C=5$.

\begin{tabular}{|l|l|c|c|c|c|c|c|}
\hline$k$ & DoF & $e\left(u_{h}\right)$ & $C V_{\text {error }}$ & $\left\|G u_{h}-\nabla u\right\|$ & $C V_{\text {recov }}$ & $\frac{\left\|G^{*} u_{h}-\nabla u\right\|}{e\left(u_{h}\right)}$ & $\frac{\eta\left(u_{h}\right)}{e\left(u_{h}\right)}$ \\
\hline 1 & 384 & $3.06 \mathrm{E}-01$ & & $3.00 \mathrm{E}-01$ & & $9.99 \mathrm{E}-01$ & 1.85 \\
\hline 8 & 1812 & $9.72 \mathrm{E}-02$ & 1.47 & $6.95 \mathrm{E}-02$ & 1.88 & $1.00 \mathrm{E}+00$ & 1.76 \\
\hline 12 & 7254 & $4.75 \mathrm{E}-02$ & 1.03 & $2.41 \mathrm{E}-02$ & 1.52 & $9.33 \mathrm{E}-01$ & 1.75 \\
\hline 34 & 49503 & $1.78 \mathrm{E}-02$ & 1.02 & $6.80 \mathrm{E}-03$ & 1.31 & $9.33 \mathrm{E}-01$ & 1.75 \\
\hline 64 & 201411 & $8.89 \mathrm{E}-03$ & 0.99 & $2.90 \mathrm{E}-03$ & 1.21 & $9.36 \mathrm{E}-01$ & 1.75 \\
\hline
\end{tabular}

Table 3: Discontinuous coefficient $a$ : $C=5, \gamma=20$, singular solution (local refinement).
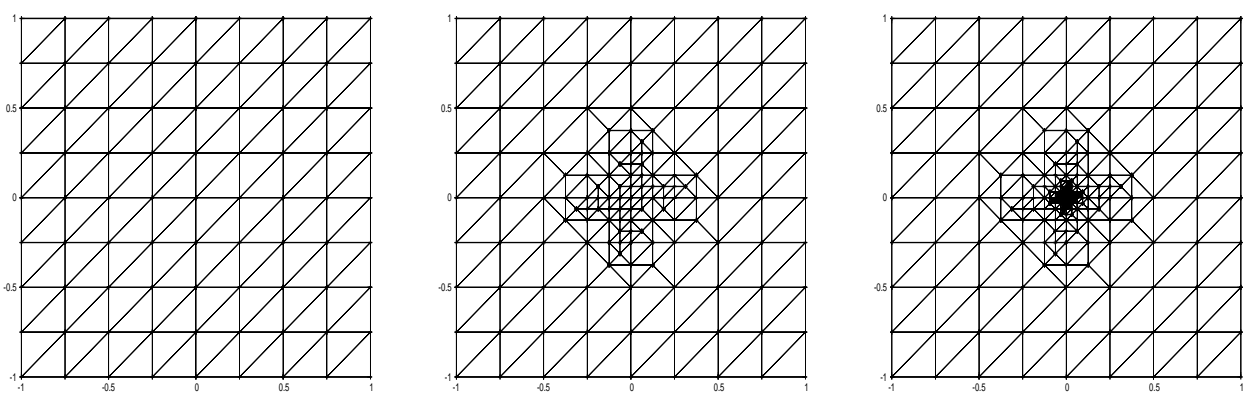

Figure 4: Mesh levels 1, 3 and 10, singular solution for $C=100$. 


\begin{tabular}{|l|l|c|c|c|c|c|c|}
\hline$k$ & DoF & $e\left(u_{h}\right)$ & $C V_{\text {error }}$ & $\left\|G u_{h}-\nabla u\right\|$ & $C V_{\text {recov }}$ & $\frac{\left\|G^{*} u_{h}-\nabla u\right\|}{e\left(u_{h}\right)}$ & $\frac{\eta\left(u_{h}\right)}{e\left(u_{h}\right)}$ \\
\hline 1 & 384 & $3.39 \mathrm{E}-01$ & & $3.38 \mathrm{E}-01$ & & $9.99 \mathrm{E}-01$ & 4.10 \\
\hline 3 & 1596 & $3.16 \mathrm{E}-01$ & 0.10 & $3.13 \mathrm{E}-01$ & 0.11 & $9.99 \mathrm{E}-01$ & 4.07 \\
\hline 12 & 6996 & $1.81 \mathrm{E}-01$ & 0.75 & $1.74 \mathrm{E}-01$ & 0.80 & $9.97 \mathrm{E}-01$ & 4.01 \\
\hline 25 & 36054 & $6.88 \mathrm{E}-02$ & 1.17 & $6.20 \mathrm{E}-02$ & 1.26 & $1.02 \mathrm{E}+00$ & 3.68 \\
\hline 38 & 123642 & $3.16 \mathrm{E}-02$ & 1.26 & $2.41 \mathrm{E}-02$ & 1.53 & $1.29 \mathrm{E}+00$ & 2.82 \\
\hline
\end{tabular}

Table 4: Discontinuous coefficient $a$ : $C=100, \gamma=500$, singular solution (local refinement). 


\section{References}

[1] M. Ainsworth. A posteriori error estimation for discontinuous Galerkin finite element approximation. SIAM J. Numer. Anal., 45(4):1777-1798 (electronic), 2007.

[2] M. Ainsworth and J. Oden. A posteriori error estimation in finite element analysis. John Wiley and Sons, 2000.

[3] D. G. Arnold, F. Brezzi, B. Cockburn, and L. D. Marini. Unified analysis of discontinuous Galerkin methods for elliptic problems. SIAM J. Numer. Anal., 39:1749-1779, 2001.

[4] I. Babuška and T. Strouboulis. The finite element methods and its reliability. Clarendon Press, Oxford.

[5] R. Becker, P. Hansbo, and M. G. Larson. Energy norm a posteriori error estimation for discontinuous Galerkin methods. Comput. Meth. Appl. Mech. Engrg., 192:723-733, 2003.

[6] D. Braess and J. Schöberl. Equilibrated residual error estimator for edge elements. Math. Comp., 77(262):651-672, 2008.

[7] S. C. Brenner and L. R. Scott. The Mathematical Theory of Finite Element Methods. Springer Verlag, New York, 1994.

[8] P. G. Ciarlet. The finite element method for elliptic problems. North-Holland, Amsterdam, 1978.

[9] S. Cochez-Dhondt and S. Nicaise. Equilibrated error estimators for discontinuous Galerkin methods. Numer. Meth. PDE, 24:1236-1252, 2008.

[10] B. Cockburn, G. E. Karniadakis, and C.-W. Shu. The development of discontinuous Galerkin methods, volume 11 of Lect. Notes Comput. Sci. Eng. Springer Verlag, Berlin, 2000 .

[11] M. Costabel, M. Dauge, and S. Nicaise. Singularities of Maxwell interface problems. RAIRO Modél. Math. Anal. Numér., 33:627-649, 1999.

[12] E. Creusé and S. Nicaise. Anisotropic a posteriori error estimation for the mixed discontinuous Galerkin approximation of the Stokes problem. Numer. Meth. PDE, 22:449-483, 2006.

[13] E. Dari, R. Durán, C. Padra, and V. Vampa. A posteriori error estimators for nonconforming finite element methods. M2AN, 30:385-400, 1996.

[14] A. Ern, S. Nicaise, and M. Vohralík. An accurate $\mathbf{H}($ div) flux reconstruction for discontinuous Galerkin approximations of elliptic problems. C. R. Math. Acad. Sci. Paris, 345(12):709-712, 2007. 
[15] A. Ern, A. F. Stephansen, and M. Vohralík. Guaranteed and robust discontinuous galerkin a posteriori error estimates for convection-diffusion-reaction problems. JCAM. to appear.

[16] F. Fierro and A. Veeser. A posteriori error estimators, gradient recovery by averaging, and superconvergence. Numer. Math., 103(2):267-298, 2006.

[17] P. Houston, I. Perugia, and D. Schötzau. Energy norm a posteriori error estimation for mixed discontinuous Galerkin approximations of the Maxwell operator. Comput. Meth. Appl. Mech. Engrg., 194:499-510, 2005.

[18] P. Houston, I. Perugia, and D. Schötzau. A posteriori error estimation for discontinuous Galerkin discretization of the $\mathrm{H}(\mathrm{curl})$-ellipic partial differential operator. IMA J. Numer. Analysis, 27:122-150, 2007.

[19] W. Hoffmann, A.H. Schatz, L.B. Wahlbin and G. Wittum. Asymptotically exact a posteriori estimators for the pointwise gradient error on each element in irregular meshes, Part I : A smooth problem and globally quasi-uniform meshes. Math. Comp., $70: 897-909,2001$.

[20] O. A. Karakashian and F. Pascal. A posteriori error estimates for a discontinuous Galerkin approximation of second-order problems. SIAM J. Numer. Anal., 41:23742399, 2003.

[21] P. Ladevèze and D. Leguillon. Error estimate procedure in the finite element method and applications. SIAM J. Numer. Anal., 20:485-509, 1983.

[22] R. Lazarov, S. Repin, and S. Tomar. Functional a posteriori error estimates for discontinuous galerkin approximations of elliptic problems. Report 2006-40, Ricam, Austria, 2006.

[23] R. Luce and B. Wohlmuth. A local a posteriori error estimator based on equilibrated fluxes. SIAM J. Numer. Anal., 42:1394-1414, 2004.

[24] P. Monk. A posteriori error indicators for Maxwell's equations. J. Comput. Appl. Math., 100:73-190, 1998.

[25] P. Monk. Finite element methods for Maxwell's equations. Numerical Mathematics and Scientific Computation. Oxford University Press, 2003.

[26] P. Neittaanmaäki and S. Repin. Reliable methods for computer simulation: error control and a posteriori error estimates., volume 33 of Studies in Mathematics and its applications. Elsevier, Amsterdam, 2004.

[27] S. Nicaise, K. Witowski, and B. I. Wohlmuth. An a posteriori error estimator for the Lamé equation based on equilibrated fluxes. IMA J. Numer. Anal., 28(2):331-353, 2008. 
[28] B. Rivière and M. Wheeler. A posteriori error estimates for a discontinuous Galerkin method applied to elliptic problems. Comput. Math. Appl., 46(1):141-163, 2003.

[29] A. Schmidt and K. G. Siebert. Design of adaptive finite element software, volume 42 of Lecture Notes in Computational Science and Engineering. Springer-Verlag, Berlin, 2005. The finite element toolbox ALBERTA, With 1 CD-ROM (Unix/Linux).

[30] K. Shahbazi. An explicit expression for the penalty parameter of the interior penalty method. J. Comput. Phys., 205:401-407, 2005.

[31] S. Sun and M. F. Wheeler. $L^{2}\left(H^{1}\right)$ norm a posteriori error estimation for discontinuous Galerkin approximations of reactive transport problems. J. Sci. Comput., 22-23:501530, 2005.

[32] A.H. Schatz and L.B. Wahlbin. Asymptotically exact a posteriori estimators for the pointwise gradient error on each element in irregular meshes, Part II : The piecewise linear case. Math. Comp., 73:517-523, 2004.

[33] R. Verfurth. A review of a posteriori error estimation and adaptive mesh-refinement techniques. Teubner Skripten zur Numerik, 1996.

[34] T. Warburton and J. S. Hesthaven. On the constants in $h p$-finite element trace inverse inequalities. Comput. Methods Appl. Mech. Engrg., 192:2765-2773, 2003.

[35] Z. Zhang. A posteriori error estimates on irregular grids based on gradient recovery. Adv. Comput. Math., 15 : 363-374, 2001. 